\title{
Mirror Therapy in the Treatment of the Phantom Pain - Case Report
}

\author{
Anita Stankovic* \\ Doctor of medical science, PMR specialist Clinic of physical medicine and rehabilitation, Serbia
}

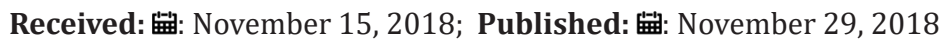

*Corresponding author: Anita Stankovic, Doctor of medical science, PMR specialist Clinic of physical medicine and rehabilitation, Serbia

\begin{abstract}
Phantom pain, pain that patients feel in the place when missing limb would be, occurs in 50-85\% of amputees. It is a known fact that mirror therapy can help in treatment of this unbearable problem. This case study presents a patient, who is insulin dependent diabetic, extremely obese, limited in her day to day activities and practically immobile. Above the knee right leg amputation was done due to severe skin infection of the right foot and shin. Taking her weight into consideration ( $>135 \mathrm{~kg}$ ), special reinforced prosthesis was made. During the rehabilitation she abruptly developed a severe pain in the missing limb, which she described as a hard, deep, and pressure like. We tried to manage it with pain killers, which did not have any impact on the intensity of the pain. The patient's condition was accompanied by symptoms of depression - crying, sadness, insomnia, hopelessness. Since symptoms have persisted, mirror therapy was included. In the assessment of the pain intensity, a numerical scale of pain was used (no pain 0- unbearable pain 10). PHQ-9 and BDI were used to evaluate depressive behaviors. Every day for three weeks the mirror has been placed in that manner, so that she sees the existing leg in it as hers missing contralateral limb. She also performed a light 20 minutes exercise. Before the therapy, the patient assessed the intensity of the pain with 9 , after one week pain was reduced to 4 , and after three weeks it was graded with 1. Symptoms of depression followed the pain reduction. At the end of her hospital rehabilitation she was advised to continue the therapy at home. Mirror therapy proved to be simple, effective and inexpensive solution for phantom limb pain in this particular case.
\end{abstract}

Keywords: Mirror Therapy; Pain; Amputation

\section{Introduction}

Phantom pain, pain that patients feel in the missing limb, occurs in $50-85 \%$ of amputees. It is most common in the first days after amputation [1]. This kind of pain can be the consequence of the discrepancy between visual feedback and the proprioceptive representation of the amputated limb [2]. The effect that mirror therapy has to phantom pain, has not been fully clarified. The positive effect of this therapy is attributed to neuron activation in the large-brain contralateral hemisphere, which looses their function of registering the missing limb movement. Visualization of the motion in the mirror could help reorganization of the neuron receptors and reintegration of the amputated extremity presentation, using socalled mirror neurons (Rizzolatti), in which way conflict between motor intent, proprioception and visualization of the amputated limb is resolved [3].

Case Report - Patient DZ. Female. Born in Nis, Serbia, in 1951. The patient is insulin dependent diabetic of 20 years, and also extremely obese $(>135 \mathrm{~kg}$ ). She is very limited in her day to day activities and practically immobile. Permanent urinal catheter due to immobility and obesity was inevitable. Above the knee right leg amputation was done on August 9th, 2017. due to severe skin infection of the right foot and shin. Postoperative course was without complications. Rehabilitation treatment was carried out at the Clinic for Physical Medicine, Rehabilitation and Prosthetics, Clinical Center Nis, Serbia, in two stages. First hospitalization took place from September 9th until November 8th, 2017. During that time, she was examined by cardiologist and vascular surgeon in order for her cardiovascular status and general health to be established. After that the specialist for prosthetics was included. He took measurements and it was clear that she will need some kind of reinforcement for her prosthesis because she was overweight. During this first stage of rehabilitation she managed to stand up straight with the walking aid. PMR specialist and Physiotherapist were responsible for her overall treatment at the Clinic.

When the specially designed prosthesis was manufactured and the patient was ready to use it, which meant that she was able to stand and make a few steps with a walking stall, the rehabilitation 
treatment was continued. Second hospitalization was from January 20th up until March 19th 2017. During this time, she become capable of self-use and maintenance of the prosthesis, walking with a prosthesis and the walking aid and she managed to successfully perform everyday activities by herself. However, during this second hospitalization, a patient suddenly, during the night, experienced a severe pain in the missing limb, which she described as a hard, deep, and pressure like. We tried to manage it with usual pain killers and we also prescribed pregabalin, which did not have any impact on the intensity of the pain. The patient's condition was accompanied by symptoms of depression - crying, sadness, insomnia, hopelessness. Since symptoms have persisted, mirror therapy was included. In the assessment of the pain intensity, a numerical scale of pain was used (no pain 0- unbearable pain
10). (Table 1) PHQ-9 and BDI were used to evaluate depressive behaviors. Every day for three weeks the patient was presented with the mirror that has been placed in that manner, so that she sees the existing leg in it as her contralateral limb, at the place when the missing one was. In addition to concentrated observation of her leg in the mirror, the patient performed a light exercise of 20 minutes: movement of the foot in all directions, slight flexionextension in the knee and a hip abduction/adduction movement. Before the therapy, the patient assessed the intensity of the pain with 9, after almost every treatment, pain was reduced to 4, and after three weeks it was graded with 1 . Symptoms of depression followed the pain reduction: PHQ-9: 18-7-5. BDI: 20-12-9. At the end of her hospital rehabilitation she was advised to continue the therapy at home.

Table 1: PHQ-9 and BDI were used to evaluate depressive behaviors.

\begin{tabular}{|c|c|c|c|c|}
\hline \multicolumn{2}{|c|}{ Pain Intensity (Numerical Pain Scale 0-10) } & \multicolumn{2}{c|}{ Depresive Behaviours (Patient Health Questionnaire-9/Backs Depression Inventory) } \\
\hline \multicolumn{2}{|c|}{ NPS } & PHQ-9 & BDI & 20 \\
\hline Before Therapy & 9 & Before Therapy & 18 & 12 \\
\hline After One Week & 4 & After One Week & 7 & 9 \\
\hline After Three Weeks & 1 & After Three Weeks & 5 & \\
\hline
\end{tabular}

\section{Conclusion}

Mirror therapy in this particular case of phantom pain, proved to be a simple, painless, inexpensive and highly effective cure.

\section{References}

1. Chan B, Witt R, Charrow A, Amanda Magee, Robin Howard, et al. (2007) Mirror Therapy for Phantom Limb Pain. N Engl J Med 357: 2206-2207.
2. Rothgangel AS, Braun S, Beurskens A, Seitz, Wade DT, et al. (2011) The clinical aspects of mirror therapy in rehabilitation: a systematic review of the literature. International Journal of Rehabilitation Research 34(1): $1-13$.

3. Darnall B (2009) Self-Delivered Home Based Mirror Therapy for Lower Limb Phantom Pain. Am J Phys Med Rehabil 88(1): 78-81.

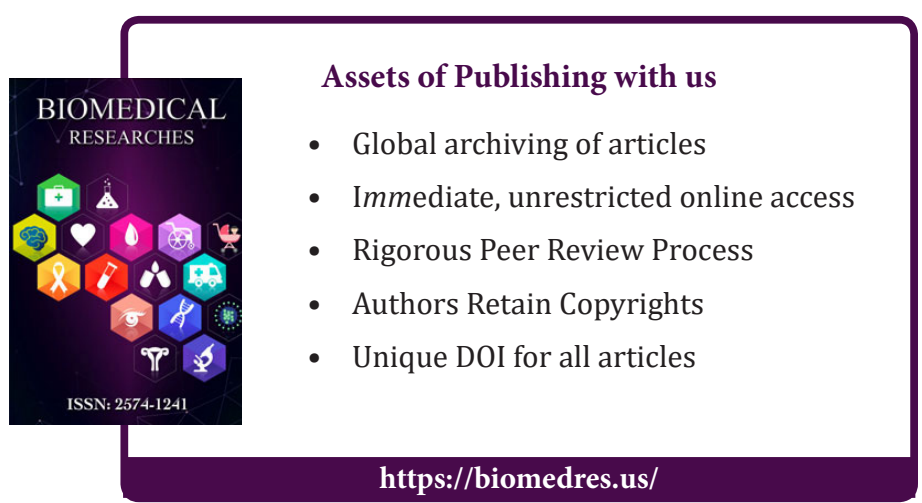

ISSN: 2574-1241

DOI: 10.26717/BJSTR.2018.11.002116

Anita Stankovic. Biomed J Sci \& Tech Res

(C) (P) This work is licensed under Creative Commons Attribution 4.0 License

Submission Link: https://biomedres.us/submit-manuscript.php 